\title{
Random Matrix Based Uncertainty Model for Complex Robotic Systems
}

\author{
Javad Sovizi ${ }^{1}$, Aliakbar Alamdari ${ }^{2}$, Sonjoy Das ${ }^{3}$ and Venkat Krovi ${ }^{4}$
}

\begin{abstract}
In this paper, we generalize our random matrix based (RM-based) uncertainty model for manipulator Jacobian matrix to the dynamic model of the robotic systems. Conventional random variable based ( $\mathrm{RV}$-based) schemes require a detailed knowledge of the system parameters variation and may be not able to fully characterize the uncertainties of the complex dynamic systems. However, the proposed RM-based approach provides a probabilistic framework for systematic characterization of the uncertainties in the complex systems with limited available information. Moreover, RM-based uncertainty model is an efficient mathematical tool that ensures the kinematic and dynamic consistency and takes into account the system complexity, configuration, structural inter-dependencies, etc. The application of the RM-based uncertainty model is investigated using an example of kinematically redundant planar parallel manipulator (3-(P)RRR). The simulation results are compared with those obtained through conventional RV-based approach and the effectiveness of the proposed method is discussed.
\end{abstract}

\section{INTRODUCTION}

The topic of uncertainties in the stochastic dynamic systems has been widely investigated over the past decades especially with the focus on the estimation and control problems. However, techniques that address the uncertainty characterization and subsequently design of the systems with lower level of the uncertainties (or, in the other words, less sensitive to the uncertainties) have not received as much attention. In a general classification, the system uncertainties can be categorized into aleatory and epistemic [1]. The former, as the name suggests, includes inherent and irreducible variations of the system and its environment, on which the designer does not have any control. However, the latter includes the reducible uncertainties that are caused by lack of the system knowledge (and can be suppressed by increasing the state of the knowledge and collection of more data) [2], [3]. Epistemic uncertainties can be further categorized into model, phenomenological and behavioral uncertainties [2], of these, model uncertainties are induced by simplification in the construction of mathematical model that represents actual physical process. The phenomenological uncertainties are induced by the design techniques and strategies and behavioral uncertainties are associated with the human decision variations. Our goal is to capture the model and phenomenological uncertainties in a systematic manner. This is critical since the design methodology may

\footnotetext{
${ }^{1}$ Javad Sovizi is with Mechanical Engineering Department, University at Buffalo, Buffalo, NY, USA. javadsovebuffalo. edu

${ }^{2}$ Aliakbar Alamdari is with Mechanical Engineering Department, University at Buffalo, Buffalo, NY, USA. aalamdar@buffalo. edu

${ }^{3}$ Sonjoy Das is with Faculty of Mechanical Engineering, University at Buffalo, Buffalo, NY, USA. sonjoy@buffalo. edu

${ }^{4}$ Venkat Krovi is with Faculty of Mechanical Engineering, University at Buffalo, Buffalo, NY, USA. vkrovi@buffalo.edu
}

actually produce the uncertainties (or magnify the effect of other variations) during system operation.

In complex dynamic systems, several parameters may be uncertain and vary over the operation time, representing stochastic processes. For example, in robotic manipulators, mass of the different parts, effective length of the links, position of the bases, etc. can be assumed as random variables at every time point, that induce disturbing (uncertain) forces (external disturbing forces are treated separately). Time varying masses, (e.g., moving platform carrying a time varying mass), deflection and bending of the links at different configurations, flexibilities at the joints and bases of the manipulators, clearances, imperfections, etc. can cause these fluctuations. However, information about the variation of all random parameters and their correlations are not known for several complex systems.

These facts necessitate a probabilistic framework that enables the uncertainty characterization in such systems that subsequently facilitates the design of systems with less fluctuations while in operation. Key requirements for an appropriate uncertainty characterization model for the robotic systems include taking into account:

- Kinematic and dynamic consistency, entire system complexity, structural inter-dependencies and intercorrelations.

- State of the system.

- System configurations, e.g., close to the singularity configurations in manipulator systems.

- Limited available information on the system fluctuations.

- Computational efficiency.

In this paper, we generalize our RM-based uncertainty model for manipulator Jacobian matrix, developed in [4], [5], that is based on the non-parametric probabilistic model proposed by Soize [6], [7], to dynamic equations of motion (EOM) of the robotic systems. This provides a systematic approach that adequately satisfies the requirements noted above and can be beneficial in the design procedure of the complex dynamic systems (from the uncertainty perspective). The system matrices (inertial mass, Coriolis \& centripetal and input transformation) are considered as random matrices and using the maximum entropy principle an appropriate probability model is constructed, i.e., a matrix-variate density function is derived.

The remainder of this paper is structured as follows. Section II concerns the derivation of the random matrix formulation of the dynamic system matrices. Section III describes the dynamic EOM of a kinematically redundant planar parallel manipulator that is used as a case study for 
the developed method. The simulations and results based on the conventional random variable scheme and the approach proposed in this paper are presented and compared in section IV. A brief discussion and directions of the future work are provided in the last section.

\section{RANDOM MATRIX FORMULATION}

In this section, our random matrix formulation of the manipulator Jacobian matrix developed in [4], [5] is generalized to dynamic EOM of the robotic systems. The EOM of the deterministic mean dynamic system can be expressed as:

$$
\underline{\mathrm{M}}(q) \ddot{q}+\underline{\mathrm{C}}(q, \dot{q}) \dot{q}+\underline{\mathrm{N}}(q)=\underline{\mathrm{E}}(q) \tau
$$

in which under-bar represents the mean value that will be defined shortly, $q=\left[\begin{array}{llll}q_{1} & q_{2} & \ldots & q_{n}\end{array}\right]^{T}$ is the system state vector, $\underline{\mathrm{M}}(q) \in \mathbb{M}_{n}^{+}$is inertial mass matrix $\left(\mathbb{M}_{n}^{+}\right.$denotes the space of $n \times n$ symmetric positive definite matrices), $\underline{\mathrm{C}}(q, \dot{q}) \in \mathbb{M}_{n}$ is the Coriolis and centripetal matrix $\left(\mathbb{M}_{n}\right.$ denotes the space of real $n \times n$ matrices), $\underline{\mathrm{N}}(q) \in \mathbb{R}^{n \times 1}$ is the gravitational force vector, $\underline{\mathrm{E}}(q) \in \mathbb{M}_{n}$ is the input transformation matrix and $\tau \in \mathbb{R}^{n \times 1}$ is the input to the system. As motivated in the preceding section, we model the uncertainties in the system through formulation of the system matrices as random matrices. The stochastic counterpart of the deterministic mean system described in Eq. (1) can be written as:

$$
\mathbf{M} \ddot{q}+\mathbf{C} \dot{q}+\mathbf{N}=\mathbf{E} \tau
$$

In Eq. (1) and (2) and forthcoming equations, capital letters are used for matrix notation and bold letters indicate the random vector or matrix. Note that the system matrices are nonlinear functions of the system state $(q)$. So, let us make the following assumption.

Assumption 1: The state of the system at each time point, $q_{k}$, can be accurately observed, however due to the uncertainties, the state at the subsequent time point $\mathbf{q}_{k+1}$ is random.

Now, let us assume a general symmetric and positive definite random matrix $\mathbf{A}$. The main goal is to construct a probability model for $\mathbf{A}$, i.e., deriving an appropriate matrixvariate probability density function $p_{\mathbf{A}}(A): \mathbb{M}_{n}^{+} \rightarrow \mathbb{R}$. The first step in the construction of the probability model is to identify the constraints and available information on the random matrix. Each valid density function has to satisfy the normalization constraint expressed as:

$$
\int_{\mathbf{A} \in \mathbb{M}_{n}^{+}} p_{\mathbf{A}}(A) \mathrm{d} \mathbf{A}=1
$$

In addition, we assume that the mean value of the random matrix $\mathbf{A}$, denoted by $\underline{\mathrm{A}}$, is given. So,

$$
E[\mathbf{A}]=\int_{\mathbf{A} \in \mathbb{M}_{n}^{+}} \mathbf{A} p_{\mathbf{A}}(A) \mathrm{d} \mathbf{A}=\underline{A}
$$

To find $p_{\mathbf{A}}(A)$, an information-theoretic approach is employed. In this approach, the density function is obtained using maximum entropy principle proposed by Jaynes [8], i.e., the entropy measure, initially introduced by Shannon [9], is maximized subjected to the existing constraints. In fact, maximizing the entropy measure of uncertainty helps to pick from amongst the set of all admissible density functions (that satisfy the prior information and constraints). This method has been frequently used to obtain the density function of the systems random variables e.g., Soize [6], [7], [10], Adhikari [11] and Ghanem and Das [12] used the maximum entropy method to derive the probability model of the structural system matrices (mass, damping, stiffness and frequency response function matrices). Das and Ghanem [13], [14] further addressed this problem by incorporating additional upper and lower bound constraints on the system matrices. A similar approach is followed here to find $p_{\mathbf{A}}(A)$. Entropy measure for density function $p_{\mathbf{A}}(A)$ is defined as:

$$
S(p)=-\int_{\mathbf{A} \in \mathbb{M}_{n}^{+}} p_{\mathbf{A}}(A) \ln \left\{p_{\mathbf{A}}(A)\right\} \mathrm{d} \mathbf{A}
$$

The Lagrangian multiplier method can be used to maximize $S(p)$ subjected to the constraints in Eq. (3) and (4). The Lagrangian functional is constructed as:

$$
\begin{aligned}
& \mathscr{L}\left(p_{\mathbf{A}}(A)\right)=-\int_{\mathbf{A} \in \mathbb{M}_{n}^{+}} p_{\mathbf{A}}(A) \ln \left\{p_{\mathbf{A}}(A)\right\} \mathrm{d} \mathbf{A} \\
& -\left(\lambda_{0}-1\right)\left(\int_{\mathbf{A} \in \mathbb{M}_{n}^{+}} p_{\mathbf{A}}(A) \mathrm{d} \mathbf{A}-1\right) \\
& -\operatorname{tr}\left[\Lambda_{1}\left(\int_{\mathbf{A} \in \mathbb{M}_{n}^{+}} \mathbf{A} p_{\mathbf{A}}(A) \mathrm{d} \mathbf{A}-\underline{A}\right)\right]
\end{aligned}
$$

where $\left(\lambda_{0}-1\right) \in \mathbb{R}$ and $\Lambda_{1} \in \mathbb{M}_{n}^{+}$are Lagrange multipliers corresponding to the normalization and mean constraints, respectively. Using the variational and matrix calculus it can be shown that (see [11])

$$
p_{\mathbf{A}}(A)=\frac{r^{n r} \operatorname{det}(\underline{A})^{-r}}{\Gamma_{n}(r)} \operatorname{etr}\left\{-r \underline{A}^{-1} A\right\} \quad \text { and } \quad r=\frac{1}{2}(n+1)
$$

where $\operatorname{etr}()=.\exp \{\operatorname{trace}()$.$\} and \Gamma_{m}(a)$ is the multivariate gamma function defined as [15]:

$$
\Gamma_{m}(a)=\pi^{\frac{1}{4} m(m-1)} \prod_{i=1}^{m} \Gamma\left[a-\frac{1}{2}(i-1)\right]
$$

where $\operatorname{Re}(a)>\frac{1}{2}(m-1)$ and $\Gamma(u)$ is the univariate gamma function. Comparing the obtained density function described by Eq. (7) with the well-known matrix-variate Wishart distribution, given in Eq. (9), verifies that the derived density function $p_{\mathbf{A}}(A)$ is a Wishart distribution. An $n \times n$ random symmetric positive definite matrix $\mathbf{S}$ is said to have a Wishart distribution with parameters $n, p$ and $\Sigma \in \mathbb{M}_{n}^{+}$, denoted by $\mathbf{S} \sim W(n, p, \Sigma)$, if its probability density function (pdf) is given by [15]:

$$
\begin{gathered}
\left.p_{\mathbf{S}}(S)=\left\{2^{\frac{1}{2} p n} \Gamma_{n}\left(\frac{1}{2} p\right) \operatorname{det}(\Sigma)^{\frac{1}{2} p}\right)\right\}^{-1} \\
\operatorname{det}(S)^{\frac{1}{2}(p-n-1)} \operatorname{etr}\left(-\frac{1}{2} \Sigma^{-1} S\right)
\end{gathered}
$$

where $S \in \mathbb{M}_{n}^{+}$and $p \geq n$.

Comparing Eq. (7) with Eq. (9) shows that $\mathbf{A}$ has a Wishart distribution with parameters $n, p=n+1$ and $\Sigma=\frac{\underline{A}}{n+1}$, i.e., $\mathbf{A} \sim W\left(n, n+1, \frac{\underline{A}}{n+1}\right)$. 
While $p_{\mathbf{A}}(A)$ is derived, more steps are required to fully realize the final proper density function for A. Eq. (3) and (4) are the only constraints used in the Lagrangian formulation (Eq. (6)). So, the resultant density function given in Eq. (7) is maximally uncertain [11]. Existence of the inverse moments is the additional constraint that we may utilize in the maximization of the entropy measure. Though $\mathbf{A} \in \mathbb{M}_{n}^{+}$implies the existence of the $\mathbf{A}^{-1}$ but it does not entail the existence of the moments of $\mathbf{A}^{-1}$. In fact, the inverse moment constraint requires $E\left[\left\|\mathbf{A}^{-1}\right\|_{\mathrm{F}}^{v}\right]<\infty$ where \|\|$_{\mathrm{F}}$ is the Frobenius norm and $v>1$ is an integer denoting the order of the moment. It is shown [6], [11] that this constraint can be equivalently written as $E\left[\ln \left(\operatorname{det}(\mathbf{A})^{-v}\right)\right]<\infty$. Subsequently, the modified Lagrangian functional is:

$$
\begin{aligned}
& \mathscr{L}\left(p_{\mathbf{A}}(A)\right)=-\int_{\mathbf{A} \in \mathbb{M}_{n}^{+}} p_{\mathbf{A}}(A) \ln \left\{p_{\mathbf{A}}(A)\right\} \mathrm{d} \mathbf{A} \\
& -\left(\lambda_{0}-1\right)\left(\int_{\mathbf{A} \in \mathbb{M}_{n}^{+}} p_{\mathbf{A}}(A) \mathrm{d} \mathbf{A}-1\right) \\
& -\int_{\mathbf{A} \in \mathbb{M}_{n}^{+}} \ln \left(\operatorname{det}(\mathbf{A})^{-v}\right) p_{\mathbf{A}}(A) \mathrm{d} \mathbf{A} \\
& -\operatorname{tr}\left[\Lambda_{1}\left(\int_{\mathbf{A} \in \mathbb{M}_{n}^{+}} \mathbf{A} p_{\mathbf{A}}(A) \mathrm{d} \mathbf{A}-\underline{A}\right)\right]
\end{aligned}
$$

The density function $\left(p_{\mathbf{A}}(A)\right)$ that maximizes $\mathscr{L}\left(p_{\mathbf{A}}(A)\right)$, expressed in Eq. (10), turns out to be:

$$
p_{\mathbf{A}}(A)=\frac{r^{n r} \operatorname{det}(\underline{A})^{-r}}{\Gamma_{n}(r)} \operatorname{det}(A)^{v} \operatorname{etr}\left\{-r \underline{A}^{-1} A\right\}
$$

where $r=v+\frac{1}{2}(n+1)$. A comparison of Eq. (11) and (9) shows that $\mathbf{A} \sim W\left(n, 2 v+n+1, \frac{\underline{A}}{2 v+n+1}\right)$.

In the maximum entropy formulation, depending on the choice of the $\mathbf{A}$ or $\mathbf{A}^{-1}$ the final distribution can significantly change (information dependence). To overcome this problem, Adhikari [11], [16] further improved the obtained density function $p_{\mathbf{A}}(A)$ by so-called optimal Wishart distribution. In his approach, it is required that $E[\mathbf{A}]$ and $E\left[\mathbf{A}^{-1}\right]$ be closest to the $\underline{\mathrm{A}}$ and $\underline{\mathrm{A}}^{-1}$, respectively. His derivation suggests (see [11], [16] for the details of the derivation):

$$
\mathbf{A} \sim W\left(n, n+1+\gamma, \frac{\underline{A}}{\sqrt{\gamma(n+1+\gamma)}}\right) \text { where } \gamma=2 v
$$

To facilitate the selection of a proper order of the inverse moment $(v)$, the normalized standard deviation of $\mathbf{A}$, introduced by Soize [6], is defined as:

$$
\sigma_{\mathbf{A}}^{2}=\frac{E\left[\|\mathbf{A}-E[\mathbf{A}]\|_{\mathrm{F}}^{2}\right]}{\|E[\mathbf{A}]\|_{\mathrm{F}}^{2}}
$$

After some algebra, $\gamma$ can be derived from Eq. (13) as:

$$
\gamma=\frac{1}{\sigma_{\mathbf{A}}^{2}}\left(1+\frac{\{\operatorname{tr}(\underline{A})\}^{2}}{\operatorname{tr}\left(\underline{A}^{2}\right)}\right)-(n+1)
$$

$\sigma_{\mathbf{A}}$ is called dispersion parameter and denotes the level of the uncertainty of the random matrix $\mathbf{A}$.

Now, in order to utilize the constructed probability model in the simulation of the random dynamic system described in Eq. (2), the following general matrix factorizations are considered.

$$
\underline{\mathrm{M}}=\underline{\mathrm{M}}_{1} \underline{\mathrm{M}}_{2}, \quad \underline{\mathrm{C}}=\underline{\mathrm{C}}_{1} \underline{\mathrm{C}}_{2}, \quad \underline{\mathrm{E}}=\underline{\mathrm{E}}_{1} \underline{\mathrm{E}}_{2}
$$

and the system random matrices are modeled as:

$$
\mathbf{M}=\underline{\mathrm{M}}_{1} \mathbf{A}_{M} \underline{\mathrm{M}}_{2}, \quad \mathbf{C}=\underline{\mathrm{C}}_{1} \mathbf{A}_{C} \underline{\mathrm{C}}_{2}, \quad \mathbf{E}=\underline{\mathrm{E}}_{1} \mathbf{A}_{E} \underline{\mathrm{E}}_{2}
$$

An appropriate matrix factorization (e.g., Cholesky, QR, $\mathrm{LU}$, etc.) must be selected depending on the properties of the corresponding random matrices $(\mathbf{M}, \mathbf{C}$ and $\mathbf{E})$ as symmetry, positive definiteness, etc. For example, Soize [6], [7] used the Cholesky decomposition for the modeling of the positive definite random matrices in the structural dynamic systems.

Now, from Eq. (16) and using Eq. (15), we can simply conclude that $E\left[\mathbf{A}_{\mathbf{M}}\right]=E\left[\mathbf{A}_{\mathbf{C}}\right]=E\left[\mathbf{A}_{\mathbf{E}}\right]=I_{n}$ where $I_{n}$ is $n \times n$ identity matrix. Moreover, the dispersion parameters $\sigma_{\mathbf{A}_{\mathbf{M}}}$, $\sigma_{\mathbf{A}_{\mathbf{C}}}$ and $\sigma_{\mathbf{A}_{\mathbf{E}}}$ can be chosen based on the experience or experimental measurements.

\section{KINEMATICALLY REDUNDANT PARALLEL MANIPULATOR}

Parallel manipulators provide several advantages compared to their serial counterparts. These advantages include higher stiffness, higher accuracy, higher stability, higher payload to weight ratio, etc. However, smaller workspace, several singularity regions within their workspace and complexity of the kinematic/dynamic models are some major drawbacks associated with them. Recently, researchers have studied the kinematics and dynamics of the redundant planar parallel manipulators whose (non-redundant) basis topology is 3-RRR (underline denotes the actuated joint) parallel manipulator. For example, Ebrahimi et al. [17] introduced a 3(PR)RR redundant manipulator in which a prismatic actuator is added to all three limbs of the base manipulator. They investigated the kinematics, workspace and the singularities of the proposed 3-( $\underline{\mathrm{PR}}) \mathrm{RR}$ manipulator. In addition Kotlarski et al. [18] proposed a 3-( $\underline{\mathrm{P}} \underline{\mathrm{R} R \mathrm{R}}$ manipulator, in which the prismatic actuator is added to only one limb for singularity elimination and enlarging the workspace, and its dynamic identification was performed in another effort by Thanh et al. [19].

In this section, we review the dynamic formulation of the 3-( $\underline{P})$ RRR redundant planar parallel manipulator [19], shown in Fig. 1. The loop-closure constraints for each serial limb of the mechanism at the platform pivots are given by:

$$
f_{i}=\left[\begin{array}{c}
x_{G_{i}}+l_{i 1} \cos \left(\theta_{i}\right)+l_{i 2} \cos \left(\theta_{i}+\psi_{i}\right)-x_{E}-r_{i} \cos \left(\phi_{E}+\beta_{i}\right) \\
y_{G_{i}}+l_{i 1} \sin \left(\theta_{i}\right)+l_{i 2} \sin \left(\theta_{i}+\psi_{i}\right)-y_{E}-r_{i} \sin \left(\phi_{E}+\beta_{i}\right)
\end{array}\right]=\left[\begin{array}{l}
0 \\
0
\end{array}\right]
$$

The general EOM of the whole system shown in Fig. (1) can be written as:

$$
M(q) \ddot{q}+h(q, \dot{q})+\Phi_{q}^{T} \lambda=\tau
$$

where $q=\left[\delta, \theta_{1}, \psi_{1}, \theta_{2}, \psi_{2}, \theta_{3}, \psi_{3}, x_{E}, y_{E}, \phi_{E}\right]^{T}$ and $M(q)=\operatorname{diag}\left(M_{1}, M_{2}, M_{3}, M_{4}\right)$ is the inertial mass matrix of the system, $h(q, \dot{q})=\left[\begin{array}{lll}h_{1}^{T}, & h_{2}^{T}, h_{3}^{T}, h_{4}^{T}\end{array}\right]^{T}$ is the Coriolis and centripetal force vector, and $\tau=\left[\tau_{1}^{T}, \tau_{2}^{T}, \tau_{3}^{T}, \tau_{4}^{T}\right]^{T}$ is 
the input vector, in which $M_{i}, h_{i}$ and $\tau_{i}(i=1, \ldots, 4)$ are the mass matrix, Coriolis and centripetal force vector and external force/moment vector of the three limbs and moving platform, respectively. Differentiating $f=\left[f_{1}^{T}, f_{2}^{T}, f_{3}^{T}\right]^{T}\left(f_{i}\right.$, $(i=1, \ldots, 3)$ are given in Eq. (17)) with respect to the time yields:

$$
\Phi_{q} \dot{q}=\left[\frac{\partial f}{\partial q}\right] \dot{q}=0
$$

To eliminate the vector of Lagrangian multipliers, $\lambda$, Eq. (18) is pre-multiplied by $R^{T}$ where $R$ is the null space of the $\Phi_{q}$, i.e., $\Phi_{q} R=0$. Then,

$$
R^{T} M(q) \ddot{q}+R^{T} h(q, \dot{q})=R^{T} \tau
$$

The state vector $q$ can be decomposed into dependent $\left(q_{D}\right)$ and independent $(z)$ coordinates as $q=K\left[q_{D}^{T}, z^{T}\right]^{T}$ where $z=\left[x_{E}, y_{E}, \phi_{E}, \delta\right]^{T}, q_{D}=\left[\theta_{1}, \psi_{1}, \theta_{2}, \psi_{2}, \theta_{3}, \psi_{3}\right]^{T}$ and $K=\left[\begin{array}{ll}\frac{\partial q}{\partial q_{D}} & \frac{\partial q}{\partial z}\end{array}\right]$ is the constant permutation matrix. Then, $\dot{q}=$ $K\left[\dot{q}_{D}^{T}, \dot{z}^{T}\right]^{T}$. After some algebra, it can be shown that $\dot{q}=$ $R \dot{z}$ where $R=K\left[\begin{array}{c}-\Phi_{q_{D}}^{-1} \Phi_{z} \\ I_{4 \times 4}\end{array}\right]$, in which $I_{4 \times 4}$ is the identity matrix and $\Phi_{q_{D}}=\left[\frac{\partial f}{\partial q_{D}}\right]$ and $\Phi_{z}=\left[\frac{\partial f}{\partial z}\right]$. In Eq. (20), writing $h(q, \dot{q})=C(q, \dot{q}) \dot{q}$ and substituting $\dot{q}$ and $\ddot{q}=R \ddot{z}+\dot{R} \dot{z}$ yield the unconstrained EOM of the system in minimal (independent) coordinates as:

$$
\tilde{M} \ddot{z}+\tilde{C} \dot{z}=\tilde{E} \tau_{a}
$$

where $\tilde{M}=R^{T} M(q) R, \tilde{C}=R^{T} M(q) \dot{R}+R^{T} C(q, \dot{q}) R, \tau_{a}=$ $\left[\begin{array}{lllll}\tau_{\theta_{1}}, & \tau_{\theta_{2}}, & \tau_{\theta_{3}}, & F_{\delta}\end{array}\right]^{T}$ is the vector of the actuator forces/torques and $\tilde{E}$ is the corresponding transformation matrix. For more details on kinematic and dynamic formulation of 3-(P)RRR manipulator, the reader is referred to Refs. [18], [19], and to Refs. [20], [21] for details on the well-known methods in inverse dynamics of the constrained multibody systems.

\section{SIMULATIONS AND RESULTS}

To investigate the effectiveness of the proposed uncertainty model, in this section we perform Monte Carlo (MC) simulations based on both conventional random variable scheme and the developed random matrix method. While

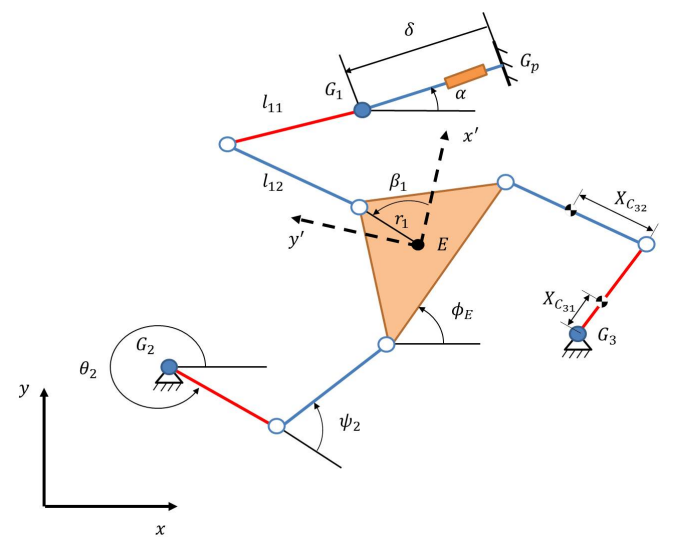

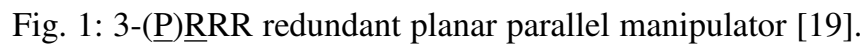

in RV-based approach, several random parameters are independently sampled and substituted in nonlinear relations at every time point, the RM-based simulation will only use a scalar dispersion parameter to generate the sample of the dynamic system matrices. As discussed above, this is very beneficial in the complex robotic systems in which limited information is available on the detailed fluctuation of the system parameters. In addition, in the real systems, the random system parameters may be not actually independent, that will increase the complexity of the sample generation in RV-based MC analysis. Moreover, in RM-based approach, sampling the system random matrices (instead of random parameters) at every time point alleviates the need of solving several nonlinear equations. In RV-based method, one may sample the elements of system matrices independently to avoid solution of these nonlinear equations, however, the resultant sample of the random matrix may be not dynamically consistent. For example, the sample of the mass matrix may be not positive definite. Furthermore, samples in RM-based analysis are drawn from well-known Wishart distribution for which efficient and accurate sampling algorithms have been developed (see for example [12]). MATLAB also provides the built-in function wishrnd for sampling from Wishart distribution. This will further facilitates the RM-based MC analysis. Despite of these advantages, there exist a question of correctness of RM-based method that will be addressed in the following subsections.

\section{A. Nominal (deterministic) System}

The initial state of the $3-(\underline{\mathrm{P}}) \underline{\mathrm{R} R R}$ manipulator, shown in Fig. 1, is set to be $z_{0}=\left[\begin{array}{llll}x_{E 0}, & y_{E_{0}}, & \phi_{E_{0}}, & \delta_{0}\end{array}\right]^{T}=$ $\left[\begin{array}{llll}0.3 & 0.3 & 0 & 0.05\end{array}\right]^{T}$. The desired task is to drive the state to $z_{\text {end }}=\left[\begin{array}{llll}0.4 & 0.4 & 0 & 0.05\end{array}\right]^{T}$ where the end effector (EE) velocity and acceleration are zero at both start and end points (rest to rest maneuver). A simple PD control scheme is used to perform this task. The control law is $\tau_{a}=(\underline{\hat{\mathrm{E}}})^{+}\left(K_{d} \dot{e}+K_{p} e\right)$ where $(.)^{+}$is the Moore-Penrose pseudo-inverse operator that is the conventional matrix inverse in cases of square matrices, $e=z_{\text {des }}-z$ is the error vector that implies $\dot{e}=$ $\dot{z}_{d e s}-\dot{z}$, and $K_{d}$ and $K_{p}$ are differential and proportional gain matrices, respectively. $K_{d}$ and $K_{p}$ are chosen to give a stable feedback control system. Here, we choose $K_{d}=5 I_{4 \times 4}$ and $K_{p}=15 I_{4 \times 4}$. The response of the system described by Eq. (21), when using this control law and nominal parameter values, is shown in Fig. 2. The nominal system specifications are also tabulated in Tab. I.

\section{B. MC Simulations}

1) RV-based MC Analysis: In this approach, at every time point, several system parameters as the mass of moving platform $\left(m_{p}\right)$, length of the links $\left(L_{i 1}\right.$ and $\left.L_{i 2}\right)$, orientation of the prismatic actuator $(\alpha)$ and position of the bases $\left(X_{G_{i}}, Y_{G_{i}}, X_{G_{p}}\right.$ and $\left.Y_{G_{p}}\right)$ are considered as random variables and their samples are generated by adding a zero-mean Gaussian perturbation to their nominal values. The nominal values of these random parameters along with the standard 
TABLE I: 3-()ㅡㄹR PM specifications; Nominal values and STD of (zero-mean) Gaussian perturbations

\begin{tabular}{|c|c|c|c|c|c|c|c|c|c|c|c|c|c|c|c|}
\hline & $\mathrm{L}_{i 1}$ & $\mathrm{~L}_{i 2}$ & $\mathrm{~m}_{i 1}$ & $\mathrm{~m}_{i 2}$ & $\mathrm{X}_{C_{i 1}}$ & $\mathrm{X}_{C_{i 2}}$ & $\mathrm{~m}_{p}$ & $\mathrm{r}_{i}$ & $\beta_{i}$ & $\mathrm{~m}_{\delta}$ & $\alpha$ & $\mathrm{X}_{G_{i}}$ & $\mathrm{Y}_{G_{i}}$ & $\mathrm{X}_{G p}$ & $\mathrm{Y}_{G_{p}}$ \\
\hline$\overline{\text { Unit }}$ & $\overline{\mathrm{m}}$ & $\overline{\mathrm{m}}$ & $\mathrm{kg}$ & $\overline{\mathrm{kg}}$ & $\bar{m}$ & $\overline{\mathrm{m}}$ & $\overline{\mathrm{kg}}$ & $\overline{\mathrm{m}}$ & $\mathrm{rad}$ & $\mathrm{kg}$ & $\overline{\mathrm{rad}}$ & $\overline{\mathrm{m}}$ & $\overline{\mathrm{m}}$ & $\overline{\mathrm{m}}$ & $\overline{\mathrm{m}}$ \\
\hline Nominal value & 0.5 & 0.6 & 0.1 & 0.1 & 0.2 & 0.3 & 1.5 & 0.107 & $\begin{array}{cc}90 & \mathrm{i}=1 \\
210 & \mathrm{i}=2 \\
330 & \mathrm{i}=3\end{array}$ & 1 & 0 & $\begin{array}{cc}0 & \mathrm{i}=2 \\
0.7 \quad \mathrm{i}=3\end{array}$ & $\begin{array}{ll}0 & \mathrm{i}=2 \\
0 & \mathrm{i}=3\end{array}$ & 0.35 & 0.7 \\
\hline $\begin{array}{c}\text { STD of } \\
\text { Gaussian perturbation }\end{array}$ & 0.01 & 0.01 & - & - & 0.01 & 0.01 & 0.05 & - & - & - & 0.1 & 0.01 & 0.01 & 0.01 & 0.01 \\
\hline
\end{tabular}
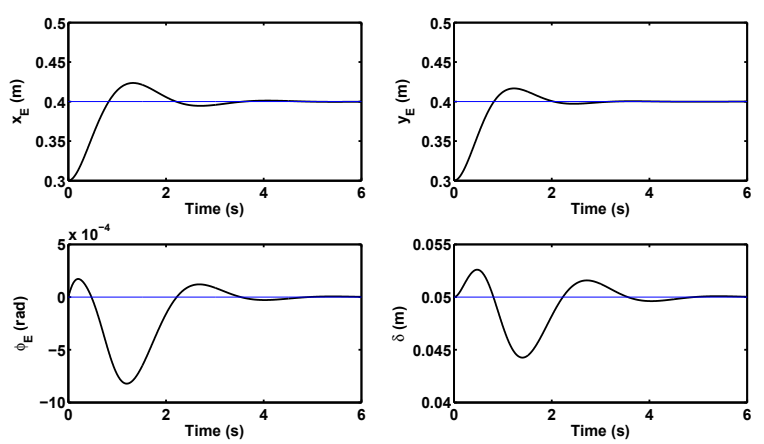

Fig. 2: Response of the nominal (deterministic) system

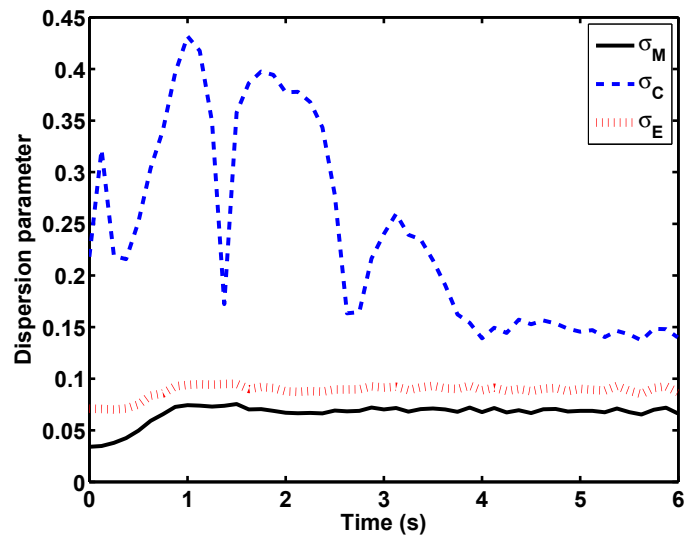

Fig. 3: Time evolution of the dispersion parameters

deviation (STD) of the corresponding Gaussian perturbations are summarized in Tab. I.

Following this procedure, 500 simulations are performed for $t \in[0,6] \mathrm{s}$ (with time step size $\Delta t=0.005 \mathrm{~s}$ ) and the realizations of the system response $\left(x_{E}, y_{E}\right.$ and $\left.\phi_{E}\right)$ are obtained for statistical analysis. In addition, to perform a similar analysis with nearly the same level of uncertainty in the RM-based approach, we obtained the dispersion parameters of the system matrices (using their samples constructed by substituting the samples of the system random parameters) at different time points using Eq. (13). Fig. 3 shows the time evolution of the dispersion parameters corresponding to $\tilde{\mathbf{M}}$, $\tilde{\mathbf{C}}$ and $\tilde{\mathbf{E}}$.

2) RM-based MC Analysis: Using the dispersion parameters (the only information needed for RM-based method), obtained as described in section IV-B.1, we are able to perform RM-based MC analysis (with approximately the same level of uncertainty) as follows. At each time point, the mean matrices are calculated by substituting the nominal values of the parameters and accurately observed state (assumption 1). Subsequently, obtained mean matrices are decomposed using a proper matrix factorization (Eq. (15)). Here, we use Cholesky decomposition for mass matrix, and LU decomposition for Coriolis and transformation matrices. Then, using the constructed probability model, samples of the perturbation matrices are drawn from optimal Wishart distribution using Eq. (12) and (14). Substituting the samples in Eq. (16) generates a sample of the $\tilde{\mathbf{M}}, \tilde{\mathbf{C}}$ and $\tilde{\mathbf{E}}$ at the corresponding time point and the system is solved for the subsequent time step toward the end of the simulation time. Using this procedure, we performed $500 \mathrm{MC}$ simulations and, similar to the RV-based analysis, the realizations of $x_{E}$, $y_{E}$ and $\phi_{E}$ are obtained for the statistical analysis.

3) Comparison: Fig. 4 shows the mean and STD of the EE position and orientation error (denoted by $e_{X}$ and $e_{\phi}$, respectively) versus the time for both RV-based and RM-based analyses. From the realizations of the $x_{E}(t)$, $y_{E}(t)$ and $\phi_{E}(t), e_{X}$ and $e_{\phi}$ are obtained as $e_{X}(t)=$ $\sqrt{\left(x_{E}(t)-x_{E}^{d}\right)^{2}+\left(y_{E}(t)-y_{E}^{d}\right)^{2}}$ and $e_{\phi}(t)=\left|\phi_{E}(t)-\phi_{E}^{d}\right|$ where $x_{E}^{d}=0.4 \mathrm{~m}, y_{E}^{d}=0.4 \mathrm{~m}$ and $\phi_{E}^{d}=0 \mathrm{rad}$ are desired EE $x$-position, $y$-position and orientation, respectively.

From Fig. 4, it can be seen that RM-based results are in good agreement with those obtained from RV-based analysis. The mean position and orientation errors corresponding to RM-based analysis (dotted red lines) are closely following the mean error trajectories obtained from RV-based method (solid black lines). Fig. 4 also shows that in both position and orientation error cases, the RM-based STD trajectory has similar trend (over the simulation time) to that of the RV-based approach. The STD of EE position error obtained from RM-based analysis is only slightly higher than the STD corresponding to RV-based method. This difference is relatively more for orientation error. As noted above, there are some unknown (and unmodeled) uncertainties that are taken into account by RM-based approach. For example, perturbing the random parameters does not perturb the zero components in the system matrices, i.e., does not include any variation due to unmodeled coupling in the system, while perturbation of the random matrices incorporates such coupling effects. This can result in higher level of variations in the system response.

\section{DISCUSSION}

In this paper, we generalized our RM-based probability model of the manipulator Jacobian matrix [4], [5], that is based on the non-parametric probabilistic formulation proposed by Soize [6], [7], to the dynamic equations of motion (EOM) of the robotic systems. The main objective is to provide a systematic approach in a probabilistic framework for the uncertainty characterization in complex dynamic systems. Conventional RV-based approaches are unable to fully 


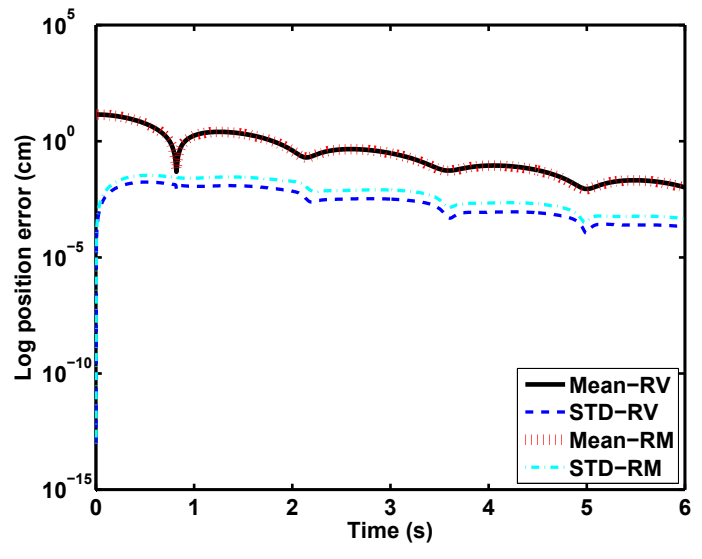

(a)

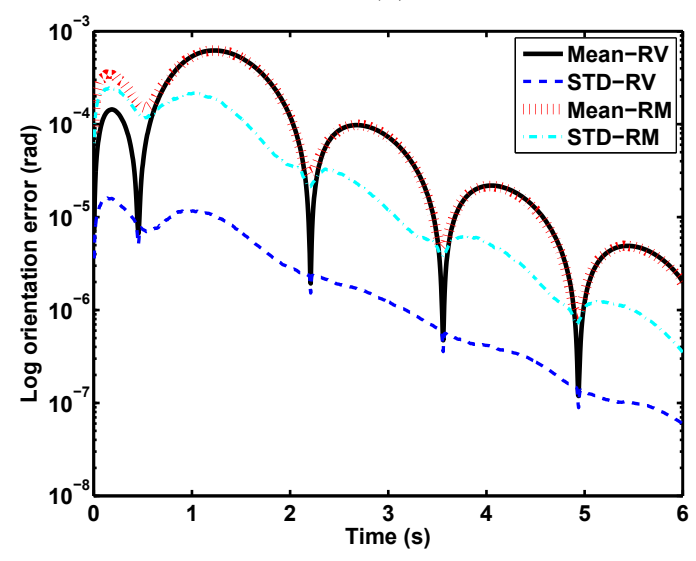

(b)

Fig. 4: Mean and STD of the EE position and orientation errors corresponding to RV-based and RM-based approaches

characterize the uncertainties for several complex systems due to the structural inter-dependencies and correlations, couplings, unknown modeling errors and generally limited available information on the uncertainties in these systems. However, the RM-based approach provides an appropriate tool for quantification of the uncertainties that is well-suited for complex robotic systems. The constructed probability model only requires a scalar dispersion parameter that can be obtained based on the experience or from the experiments. In addition, it adequately satisfies some requirements of an appropriate probability model for robotic systems, as accounting for the system state and configuration (e.g., close to singularity regions), computational efficiency, etc. To examine the proposed method, we performed MC analysis on the dynamic model of a kinematically redundant parallel manipulator, based on the conventional random variable and proposed random matrix approaches and the results were compared to highlight the effectiveness of the developed method. It was shown that the results obtained from RMbased approach closely follow those obtained from RV-based analysis.

While we investigated the applicability of the proposed RM-based uncertainty model, however, more general conclusions can be drawn when the simulation results are compared to the experimental data. This will hopefully be included in our future studies.

\section{ACKNOWLEDGMENT}

This work was partially supported by the National Science Foundation awards IIS-1319084 and CNS-1314484.

\section{REFERENCES}

[1] J. C. Helton and D. E. Burmaster, "Treatment of aleatory and epistemic uncertainty in performance assessments for complex systems," Reliability Engineering and System Safety, vol. 54, no. 2 3, pp. 91-94, 1996.

[2] D. P. Thunnissen, "Uncertainty classification for the design and development of complex systems," in Proc. 3rd Annu. Predictive Methods Conference, Newport Beach, CA, 2003.

[3] W. Yao, X. Chen, W. Luo, M. v. Tooren and J. Guo, "Review of uncertainty-based multidisciplinary design optimization methods for aerospace vehicles," Progress in Aerospace Sciences, vol. 47, no. 6, pp. 450-479, Aug. 2011.

[4] J. Sovizi and V. N. Krovi, "Uncertainty characterization in serial and parallel manipulators using random matrix theory," in Proc. IEEE Int. Conf. Automation Science and Engineering, Wisconsin, Aug. 2013, pp. 312-317.

[5] J. Sovizi., A. Alamdari and V. N. Krovi, "A random matrix approach to manipulator Jacobian," ASME Dynamic Systems and Control Conf., CA, 2013, to be published.

[6] C. Soize, "A nonparametric model of random uncertainties for reduced matrix models in structural dynamics," Probabilistic Engineering Mechanics, vol. 15, no. 3, pp. 277-294, 2000.

[7] C. Soize, "Maximum entropy approach for modeling random uncertainties in transient elastodynamics," J. of the Acoustical Society of America, vol. 109, no. 5, pp. 1979-1996, 2001.

[8] E. T. Jaynes, "Information theory and statistical mechanics," Physical Review, vol. 106, no. 4, pp. 620-630, 1957.

[9] C. Shannon, "A mathematical theory of communication," Bell System Technical J., vol. 27, no. 3, pp. 379-423, Jul. 1948.

[10] C. Soize and H. Chebli, "Random uncertainties model in dynamic substructuring using a nonparametric probabilistic model,' J. of Engineering Mechanics, vol. 129, no. 4, pp. 449-457, 2003.

[11] S. Adhikari, "Matrix variate distributions for probabilistic structural dynamics," AIAA J., vol. 45, no. 7, pp. 1748-1762, 2007.

[12] R. Ghanem and S. Das, "Hybrid Representations of Coupled Nonparametric and Parametric Models for Dynamic Systems," AIAA J., vol. 47, no. 4, pp. 1035-1044, 2009.

[13] S. Das and R. Ghanem, "A Bounded Random Matrix Approach for Stochastic Upscaling," SIAM Multiscale Modeling and Simulation, vol. 8, no. 1, pp. 296-325, 2009.

[14] S. Das, "Model, Identification, Analysis of Complex Stochastic Systems: Applications in Stochastic Partial Differential Equations and Multiscale Mechanics," Ph.D. dissertation, Dept. Civil \& Env. Eng., Univ. of Southern California, Los Angeles, CA, 2008.

[15] A. K. Gupta and D. K. Nagar, "Matrix Variate Distributions," Boca Raton, FL: Chapman \& Hall/CRC, 2000.

[16] S. Adhikari, "Wishart Random Matrices in Probabilistic Structural Mechanics,” J. Eng. Mech., vol. 134, no. 12, pp. 1029-1044, 2008.

[17] I. Ebrahimi, J. A. Carreteroa and R. Boudreau, "3-(PR)RR redundant planar parallel manipulator: Inverse displacement, workspace and singularity analyses," J. Mech. and Mach. Theory, vol. 42, no. 8, pp. 1007-1016, 2007.

[18] J. Kotlarski, H. Abdellatif, T. Ortmaier and B. Heimann, "Enlarging the useable workspace of planar parallel robots using mechanisms of variable geometry," in Proc. ASME/IFToMM Int. Conf. Reconfigurable Mechanisms and Robots, London, United Kingdom, 2009, pp. 94-103.

[19] T. D. Thanh, J. Kotlarski, B. Heimann and T. Ortmaier, "Dynamics identification of kinematically redundant parallel robots using the direct search method," J. Mech. and Mach. Theory, vol. 52, pp. 277$295,2012$.

[20] A. Muller, "Motion Equations in Redundant Coordinates with Application to Inverse Dynamics of Constrained Mechanical Systems," Nonlinear Dynamics, vol. 67, no. 4, pp. 2527-2541, 2012.

[21] A. Laulusa and O. A. Bauchau, "Review of Classical Approaches for Constraint Enforcement in Multibody Systems," J. Comput. Nonlinear Dyn., vol. 3, 011004, 2008. 